\title{
The Effect of Imperfect Carrier Frequency Offset Estimation on OFDMA Uplink Transmission
}

\author{
Zhongshan Zhang and Chintha Tellambura \\ Department of Electrical and Computer Engineering \\ University of Alberta, Edmonton \\ AB T6G 2V4, Canada \\ \{zszhang, chintha\}@ece.ualberta.ca
}

\begin{abstract}
Since carrier frequency offset destroys user's signal orthogonality in Orthogonal Frequency-Division Multiplexing Access (OFDMA) uplink transmission, resulting in an interference-limited system, its estimation/correction is very important. The residual carrier frequency offset of each user contributes Inter-Carrier-Interference (ICI) and Multiple-UserInterference (MUI) to other users. In this paper, the effect of the carrier frequency offset on OFDMA uplink is analyzed. We first analyze the average uplink capacity losses as well as the Signal-to-Interference-and-Noise Ratio (SINR) reduction due to the carrier frequency offsets, and then discuss the capacity increases by using adaptive power allocation. The averaged Bit Error Rate (BER) performance with the carrier frequency offsets on OFDMA uplink is also analyzed.
\end{abstract}

\section{INTRODUCTION}

OFDMA (Orthogonal Frequency-Division Multiplexing Access) divides the total signal bandwidth into multiple orthogonal subcarrier groups, with each group being allocated to one user. However, in the uplink, different users have different carrier frequency offsets, resulting in an interference-limited system [1]. The carrier frequency offset of each user contributes Inter-Carrier-Interference (ICI) and Multiple-UserInterference (MUI) to other users.

It is well known that multicarrier systems such as OFDM are highly sensitive to the carrier frequency offset, and even a small frequency offset can greatly degrade their performance [2]. The BER impaired by the carrier frequency offset in OFDM is analyzed in [3]. Although several high performance classical frequency offset estimators for the OFDM downlink synchronization are available [2], [4]-[6], their performance will degrade in an interference-limited environment.

To mitigate the ICI and MUI in an OFDMA uplink, one can utilize two strategies. First, by separating subcarrier groups of different users with a large enough frequency guardband, the signal transmitted by each user can be separated successfully, as in [7]. Second, high performance frequency offset estimators, either pilot/training-sequence-based [8], [9] or not [10], are developed. A high performance Maximum Likelihood (ML) estimation of synchronization and channel estimation for the OFDMA uplink transmissions is studied in [11]. MUI cancellation in an OFDMA system is discussed in [12], where the MUI can be eliminated correctly based on the perfect knowledge of the carrier frequency offset of each user. Conventional estimators, such as [2], are considered as candidates for the frequency offset estimation for each user. Unfortunately, perfect frequency offset estimation is not achievable in any conventional algorithms, and the residual carrier frequency offset of each user degrades the interferenceeliminating performance. The reasons for the lack of perfect carrier frequency offset estimation include that perfect carrier frequency offset estimation is impractical in real systems for any statistical estimator and that the Doppler effects due to user mobility also manifest as carrier frequency offsets. Subcarrier/power allocation as well as system capacity in the multiuser OFDM/OFDMA systems are discussed in [13], [14] without considering the effect of the carrier frequency offset.

This paper analyzes the effect of the carrier frequency offsets on the OFDMA uplink transmission. Using the Channel State Information (CSI) and the statistical information of the carrier frequency offset, we derive the average SINR of each user and an adaptive power allocation scheme to improve the uplink capacity. Note that we do not consider the nearfar effect and assume the statistical character of the wireless channel of each user is identical at the receiver (base station). The Bit Error Rate (BER) of the OFDMA uplink with the carrier frequency offsets is also analyzed.

This paper is organized as follows. The OFDMA system model is described in Section II, and the received SINR for an OFDMA uplink is analyzed in Section III. The capacity of an OFDMA uplink with carrier frequency offset is derived in Section IV. A water-filling power allocation method for capacity improvement is also derived in this section. BER analysis in interference limited OFDMA uplink transmission is provided in Section V, followed by the conclusions in Section VI.

Notation: $(\cdot)^{H}$ denotes conjugate transpose. $(x)^{\dagger}=$ $\max \{x, 0\}$. The imaginary unit is $\jmath=\sqrt{-1}$. A circularly symmetric complex Gaussian variable with mean $m$ and variance $\sigma^{2}$ is denoted by $w \sim \mathcal{C N}\left(m, \sigma^{2}\right)$. The $N \times N$ allzero matrix is represented as $\mathbf{O}_{N} . \mathbb{E}\{x\}$ and $\operatorname{Var}\{x\}$ denote the mean and the variance of $x$, respectively.

\section{OFDMA Uplink SignAl MODEL}

In OFDMA systems, each user is modulated by complex data symbols from a signal constellation, e.g., phase-shift keying (PSK) or quadrature amplitude modulation (QAM). In a frequency-selective fading channel, the received signal at the 
base station can be represented as

$$
\mathbf{y}=\sum_{k} \mathbf{y}_{k}=\sum_{k}(\mathbf{E}_{k} \underbrace{\mathbf{F}_{k} \mathbf{H}_{k} \boldsymbol{\Phi}_{k} \mathbf{x}_{k}}_{\mathbf{m}_{k}}+\mathbf{w}_{k}),
$$

where $\boldsymbol{\Phi}_{k}=\operatorname{diag}\left\{\sqrt{P_{i}}: i \in G_{k}\right\}$ represents the power allocated to each subcarrier of user $k, \mathbf{H}_{k}=\operatorname{diag}\left\{H_{k}^{i}: i \in G_{k}\right\}$ with $H_{k}^{i}$ denoting the channel attenuation at the $i$-th subcarrier, and $G_{k}$ stands for the subcarriers allocated to user $k$. Note that $\bigcap_{k} G_{k}=\emptyset$ and $\bigcup_{k} G_{k} \subseteq\{0,1, \cdots, N-1\}$.

$$
\mathbf{E}_{k}=\operatorname{diag}\left\{e^{\jmath \psi_{k}}, e^{\jmath\left(\frac{2 \pi \varepsilon_{k}}{N}+\psi_{k}\right)}, \cdots, e^{\jmath\left(\frac{2 \pi \varepsilon_{k}(N-1)}{N}+\psi_{k}\right)}\right\}
$$

with $\psi_{k}$ and $\varepsilon_{k}$ representing the initial phase and the carrier frequency offset of user $k . \mathbf{F}_{k}$ denotes the Inverse Discrete Fourier Transform (IDFT) matrix for the $k$-th user (where only the subcarriers allocated to user $k$ are modulated), and $N$ is the Discrete Fourier Transform (DFT) length. In OFDMA, we have $\mathbf{F}_{k}^{H} \mathbf{F}_{l \neq k}=\mathbf{O}_{N}$. $\mathbf{x}_{k}$ is the transmit vector of user $k$, and without loss of generality, $\mathbf{x}_{k}[m]$ are Independent and Identically Distributed (IID) $\mathcal{C N}(0,1)$. We also assume that IID symbols are transmitted by each user. $\mathbf{m}_{k}=\mathbf{F}_{k} \mathbf{H}_{k} \boldsymbol{\Phi}_{k} \mathbf{x}_{k}$ represents the time-domain transmit training of user $k . \mathbf{w}_{k}$ in (1) is a vector of additive white Gaussian noise (AWGN) added to the signal space of user $k$, and that $\mathbf{w}_{k}[i] \sim \mathcal{C N}\left(0, \sigma_{w}^{2}\right)$. $\mathbf{y}$ is a complex Gaussian random vector with the Probability Density Function (PDF) given by [15]:

$$
f(\mathbf{y})=\frac{1}{\pi^{N} \operatorname{det}[\mathbf{C}]} \exp \left\{-(\mathbf{y}-\mathbf{m})^{H} \mathbf{C}^{-1}(\mathbf{y}-\mathbf{m})\right\},
$$

where $\mathbf{C}=\mathbb{E}\left\{(\mathbf{y}-\mathbf{m})(\mathbf{y}-\mathbf{m})^{H}\right\}$ and $\mathbf{m}=\sum_{\mathbf{k}} \mathbf{m}_{\mathbf{k}}$.

Carrier frequency offset in the OFDMA arises from the following three conditions:

1. Mismatch between transmit and receive oscillators.

2. Doppler Shift due to user mobility.

3. Carrier frequency offset compensation error.

Some conventional algorithms proposed for the OFDM systems (e.g., [2], [7]-[9], [11]) can also be used for carrier frequency offset estimation in an OFDMA. Uplink synchronization for each user can be performed based on the received training sequence $\mathbf{y}$. Without loss of generality, we assume that the carrier frequency offsets of different users are IID RVs. For an OFDMA system with a total of $M$ users access a base station, the Fisher Information Matrix (FIM) $\boldsymbol{\Gamma}$ is derived as

$$
[\boldsymbol{\Gamma}]_{k l}=\operatorname{trace}\left(\mathbf{C}^{-1} \frac{\partial \mathbf{C}}{\partial \varepsilon_{k}} \mathbf{C}^{-1} \frac{\partial \mathbf{C}}{\partial \varepsilon_{l}}\right) .
$$

Note that

$$
\begin{aligned}
\mathbf{C} & =\mathbb{E}\left\{\left[\sum_{k}\left(\mathbf{y}_{k}-\mathbf{m}_{k}\right)\right]\left[\sum_{k}\left(\mathbf{y}_{k}-\mathbf{m}_{k}\right)^{H}\right]\right\} \\
& =\sum_{k}\left(\mathbf{C}_{k}+\mathbf{Z}_{k}\right),
\end{aligned}
$$

where $\mathbf{C}_{k}=\mathbb{E}\left\{\left(\mathbf{y}_{k}-\mathbf{m}_{k}\right)\left(\mathbf{y}_{k}-\mathbf{m}_{k}\right)^{H}\right\}$ represents the covariance matrix of user $k$, and $\mathbf{Z}_{k l}=$ $\mathbb{E}\left\{\left(\mathbf{y}_{k}-\mathbf{m}_{k}\right)\left(\mathbf{y}_{l}-\mathbf{m}_{l}\right)^{H}\right\}$ is the MUI matrix of user $k$ that contributed by user $l$. For a non-zero carrier frequency offset for each user, the signal spaces of different users will overlap, so that $\sum_{k \neq l} \mathbf{Z}_{k l} \neq \mathbf{O}_{N}$. We can decompose $\mathbf{C}$ as

$$
\mathbf{C}=\sum_{k} \mathbf{U}\left(\mathbf{D}_{k}+\tilde{\mathbf{D}}_{k}\right) \mathbf{U}^{H}
$$

where $\mathbf{U}$ is a $N \times N$ Unitary matrix,

$$
\mathbf{D}_{k}=\operatorname{diag}\{0, \cdots, \underbrace{\lambda_{k, 1}, \cdots, \lambda_{k, \mathcal{N}_{k}}}_{G_{k}}, \cdots, 0\}
$$

with $\lambda_{k, i}$ representing the $i$-th eigenvalue of $\mathbf{C}_{k}$, and

$$
\tilde{\mathbf{D}}_{k}=\operatorname{diag}\{0, \cdots, \underbrace{z_{k, 1}, \cdots, z_{k, \mathcal{N}_{k}}}_{G_{k}}, \cdots, 0\}
$$

with $z_{k, i}$ representing the $i$-th eigenvalue of $\mathbf{Z}_{k}$. Therefore, $\mathbf{C}^{-1}$ can be represented as

$$
\mathbf{C}^{-1}=\sum_{k} \mathbf{U}\left(\mathbf{D}_{k}+\tilde{\mathbf{D}}_{k}\right)^{-1} \mathbf{U}^{H},
$$

where

$$
\left(\mathbf{D}_{k}+\tilde{\mathbf{D}}_{k}\right)^{-1}=\operatorname{diag}\{0, \cdots, \underbrace{\left(\lambda_{k, i}+z_{k, i}\right)^{-1}}_{G_{k}}, \cdots, 0\} .
$$

We also have

$$
\frac{\partial \mathbf{C}}{\partial \varepsilon_{k}}=\frac{\partial\left(\mathbf{C}_{k}+\mathbf{Z}_{k}\right)}{\partial \varepsilon_{k}}+\sum_{l \neq k} \frac{\partial\left(\mathbf{C}_{l}+\mathbf{Z}_{l}\right)}{\partial \varepsilon_{k}} .
$$

Note that $\mathbf{Z}_{k}$ is the MUI contributed by the users other than $k$ and is not a function of $\varepsilon_{k}$, so that $\frac{\partial \mathbf{Z}_{k}}{\partial \varepsilon_{k}}=\mathbf{O}_{N}$. Since $\mathbf{C}_{l}$ is also not a function of $\varepsilon_{k}$, and then $\frac{\partial \mathbf{C}_{l}}{\partial \varepsilon_{k}}=\mathbf{O}_{N}$. From the above discussion, we have

$$
\begin{aligned}
& \frac{\partial \mathbf{C}}{\partial \varepsilon_{k}}=\frac{\partial \mathbf{C}_{k}}{\partial \varepsilon_{k}}+\sum_{l \neq k} \frac{\partial \mathbf{Z}_{l}}{\partial \varepsilon_{k}}
\end{aligned}
$$

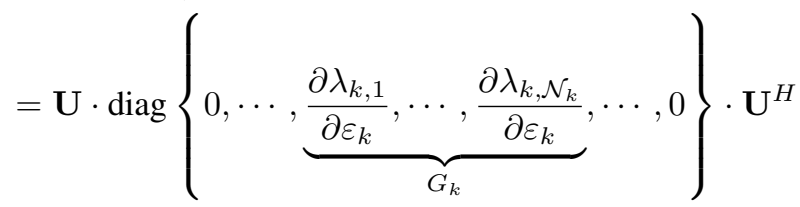

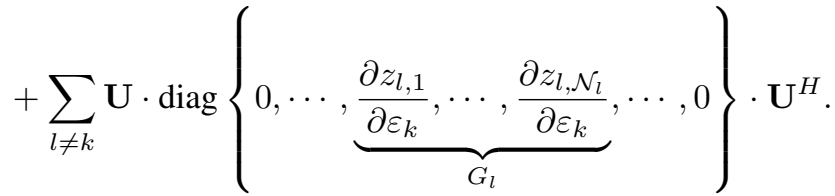




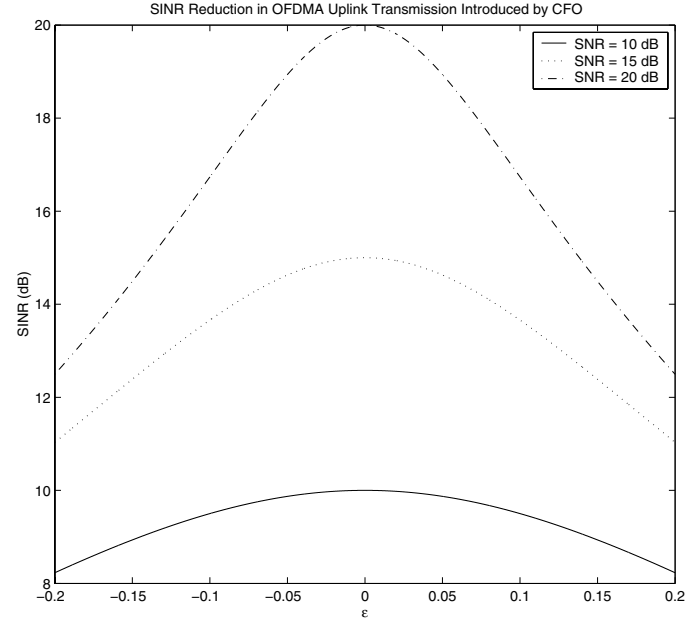

Fig. 1. SINR reduction by carrier frequency offset in OFDMA uplink transmission.

When $l \neq k$, the $k l$-th element in the FIM can be represented as

$$
\begin{aligned}
{[\boldsymbol{\Gamma}]_{k l} } & =\sum_{i=1}^{\mathcal{N}_{k}} \frac{\frac{\partial \lambda_{k, i}}{\partial \varepsilon_{k}} \frac{\partial z_{k, i}}{\partial \varepsilon_{l}}}{\left(\lambda_{k, i}+z_{k, i}\right)^{2}}+\sum_{j=1}^{\mathcal{N}_{l}} \frac{\frac{\partial \lambda_{l, j}}{\partial \varepsilon_{l}} \frac{\partial z_{l, j}}{\partial \varepsilon_{k}}}{\left(\lambda_{l, j}+z_{l, j}\right)^{2}} \\
& +\sum_{n \neq k, l} \sum_{p=1}^{\mathcal{N}_{n}} \frac{\frac{\partial^{2} z_{n, p}}{\partial \varepsilon_{k} \partial \varepsilon_{l}}}{\left(\lambda_{n, p}+z_{n, p}\right)^{2}},
\end{aligned}
$$

and the $k k$-th element in the FIM is given by

$$
[\boldsymbol{\Gamma}]_{k k}=\sum_{i=1}^{\mathcal{N}_{k}} \frac{\left(\frac{\partial \lambda_{k, i}}{\partial \varepsilon_{k}}\right)^{2}}{\left(\lambda_{k, i}+z_{k, i}\right)^{2}}+\sum_{l \neq k} \sum_{j=1}^{\mathcal{N}_{l}} \frac{\left(\frac{\partial z_{l, j}}{\partial \varepsilon_{k}}\right)^{2}}{\left(\lambda_{l, j}+z_{l, j}\right)^{2}} .
$$

From (14), for an unbiased estimator $\hat{\varepsilon}_{k}$, the CRLB is derived as

$$
\begin{aligned}
\operatorname{Var}\left\{\hat{\varepsilon}_{k}\right\} & \geq\left[\boldsymbol{\Gamma}^{-1}\right]_{k k} \geq[\boldsymbol{\Gamma}]_{k k}^{-1} \\
& =\frac{1}{\sum_{i=1}^{\mathcal{N}_{k}} \frac{\left(\frac{\partial \lambda_{k, i}}{\partial \varepsilon_{k}}\right)^{2}}{\left(\lambda_{k, i}+z_{k, i}\right)^{2}}+\sum_{l \neq k} \sum_{j=1}^{\mathcal{N}_{l}} \frac{\left(\frac{\partial z_{l, j}}{\partial \varepsilon_{k}}\right)^{2}}{\left(\lambda_{l, j}+z_{l, j}\right)^{2}}} \\
& =\frac{1}{\alpha_{k} \cdot \mathrm{SNR}_{k}^{2}}+\frac{1}{\beta_{k} \cdot \mathrm{SNR}_{k}}+\frac{1}{\varpi_{k} \cdot \mathrm{SIR}_{k}^{2}},
\end{aligned}
$$

where $\lambda_{k, i}$ and $z_{k, i}$ are defined in (7) and (8), respectively; $\alpha_{k}, \beta_{k}$, and $\varpi_{k}$ are constants specified by the structure of the training sequence used by user $k ; \mathrm{SNR}_{k}$, and $\mathrm{SIR}_{k}$ stand for the Signal-to-Noise-Ratio (SNR) and the Signal-toInterference-Ratio (SIR) of user $k$, respectively.

\section{SINR ANALYSIS}

For an OFDMA uplink transmission with all the users running at the tracking phase (carrier frequency offset acquisition has been performed, and the residual carrier frequency offset is very small), it is reasonable to assume that $\varepsilon_{k}$ for each $k$ is

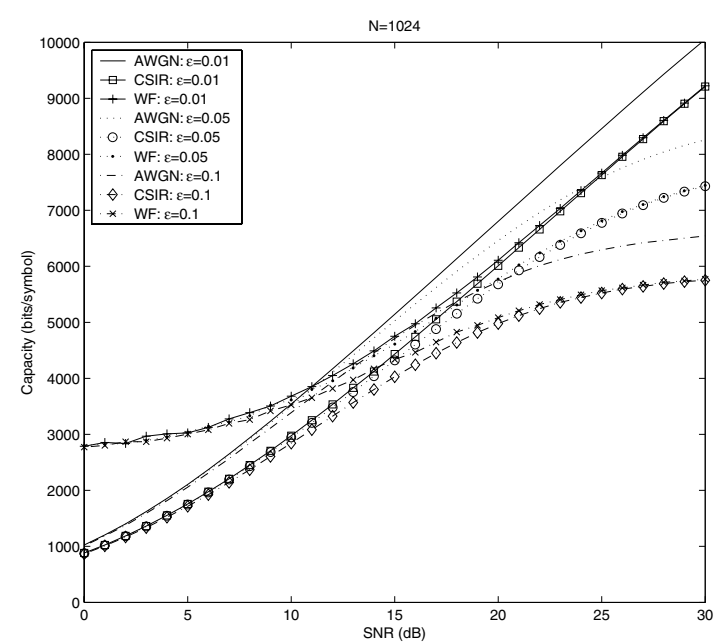

Fig. 2. OFDMA uplink capacity with non-zero carrier frequency offset.

an IID random variable that is uniformly distributed in $(-\epsilon$, $\epsilon)$ with a PDF $f_{\epsilon}\left(\varepsilon_{k}\right)=\frac{1}{2 \epsilon}$, where $\epsilon \ll 1$.

Based on the received vector $\mathbf{y}$, the averaged SINR of the $m$-th subcarrier is represented as

$$
\gamma_{m \mid m \in G_{k}}=\frac{\frac{\sin ^{2}\left(\pi \varepsilon_{k}\right)}{N^{2} \sin ^{2}\left(\frac{\pi \varepsilon_{k}}{N}\right)} \cdot\left|\sqrt{P_{m}} H_{k}^{m}\right|^{2}}{\mathbb{E}\left\{\left|\mu_{m}+\xi_{m}\right|^{2}\right\}+\sigma_{w}^{2}}
$$

with

$$
\begin{aligned}
\mu_{m} & =\sum_{i \in G_{k}, i \neq m} \sqrt{P_{i}} \mathbf{x}_{k}[i] H_{k}^{i} \frac{\sin \left[\pi\left(i-m+\varepsilon_{k}\right)\right]}{N \sin \left[\frac{\pi\left(i-m+\varepsilon_{k}\right)}{N}\right]} \\
& \cong \sum_{i \in G_{k}, i \neq m} \frac{(-1)^{i-m} \sqrt{P_{i}} \mathbf{x}_{k}[i] H_{k}^{i} \pi}{N \sin \left[\frac{\pi(i-m)}{N}\right]} \varepsilon_{k}, \\
\xi_{m} & =\sum_{l \neq k} \sum_{i \in G_{l}} \sqrt{P_{i} \mathbf{x}_{l}[i] H_{l}^{i} \frac{\sin \left[\pi\left(i-m+\varepsilon_{l}\right)\right]}{N \sin \left[\frac{\pi\left(i-m+\varepsilon_{l}\right)}{N}\right]}} \\
& \cong \sum_{l \neq k} \sum_{i \in G_{l}} \frac{(-1)^{i-m} \sqrt{P_{i}} \mathbf{x}_{l}[i] H_{l}^{i} \pi}{N \sin \left[\frac{\pi(i-m)}{N}\right]} \varepsilon_{l},
\end{aligned}
$$

where we assume that $\mathbb{E}\left\{\left|\mathbf{x}_{i}[m]\right|^{2}\right\}=\sigma_{x}^{2}=1, \sigma_{w}^{2}=\frac{\mathbb{E}\left\|\mathbf{w}_{k}\right\|^{2}}{\mathcal{N}_{k}}$, $\sin \left[\pi\left(i-m+\varepsilon_{l}\right)\right]=(-1)^{i-m} \sin \left(\pi \varepsilon_{l}\right) \cong(-1)^{i-m} \pi \varepsilon_{l}$, and $\sin \left[\frac{\pi\left(i-m+\varepsilon_{l}\right)}{N}\right] \cong \sin \left[\frac{\pi(i-m)}{N}\right]$ when $\varepsilon_{l} \ll 1$. If we further assume that $\mathbb{E}\left\{\mathbf{x}_{k}[n] \mathbf{x}_{l}^{*}[m]\right\}=0$ for $k \neq l$ or $n \neq m$, $\mathbb{E}\left\{\left|\mu_{m}+\xi_{m}\right|^{2}\right\}$ can be approximated as

$$
\begin{aligned}
\mathbb{E}\left\{\left|\mu_{m}+\xi_{m}\right|^{2}\right\} & =\frac{\pi^{2} \epsilon^{2}}{3} \sum_{i \neq m} \frac{\left|\sqrt{P_{i}} H_{l}^{i}\right|^{2}}{N^{2} \sin ^{2}\left[\frac{\pi(i-m)}{N}\right]} \\
& =\frac{\pi^{2} \epsilon^{2} \kappa_{m}}{3} \sum_{i \neq m} \frac{1}{N^{2} \sin ^{2}\left[\frac{\pi(i-m)}{N}\right]},
\end{aligned}
$$


where $\min _{i \neq m}\left\{\left|\sqrt{P_{i}} H_{l}^{i}\right|^{2}\right\} \leq \kappa_{m} \leq \max _{i \neq m}\left\{\left|\sqrt{P_{i}} H_{l}^{i}\right|^{2}\right\}$, and $\sum_{i \neq m} \frac{1}{N^{2} \sin ^{2}\left[\frac{\pi(i-m)}{N}\right]} \doteq \sum_{i \neq m} \frac{1}{\pi^{2}(i-m)^{2}} \cong \frac{1}{3}$. In this section we assume that an adaptive power allocation algorithm is performed at the transmitter, and the average received power of each user is identical. Therefore, $\gamma_{m \mid m \in G_{k}}$ can be represented as

$$
\gamma_{m \mid m \in G_{k}} \cong \frac{\mathrm{SNR}_{k}}{\frac{\pi^{2} \epsilon^{2} \mathrm{SNR}_{k}}{9}+1} \cdot \frac{\sin ^{2}\left(\pi \varepsilon_{k}\right)}{\pi^{2} \varepsilon_{k}^{2}}
$$

with $\mathrm{SNR}_{k}=\frac{\mathbb{E}\left\{\kappa_{m \mid m \in G_{k}}\right\}}{\sigma_{w}^{2}}$. The averaged SINR of user $k$ is

$$
\begin{aligned}
\operatorname{SINR}_{k} & =\int_{-\epsilon}^{\epsilon} \frac{\mathrm{SNR}_{k}}{\frac{\pi^{2} \epsilon^{2} \mathrm{SNR}_{k}}{9}+1} \cdot \frac{\sin ^{2}\left(\pi \varepsilon_{k}\right)}{\pi^{2} \varepsilon_{k}^{2}} \cdot \frac{1}{2 \epsilon} \mathrm{d} \varepsilon_{k} \\
& =\frac{\mathrm{SNR}_{k}}{\frac{\pi^{2} \epsilon^{2} \mathrm{SNR}_{k}}{9}+1} \cdot\left(1-\frac{\pi^{2} \epsilon^{2}}{9}+\frac{\pi^{4} \epsilon^{4}}{180}\right) .
\end{aligned}
$$

The SINR degradation due to the carrier frequency offsets in an OFDMA uplink transmission is shown in Fig. 1. The SINR loss increases as $\epsilon$ increases. For example, for an SNR of $20 \mathrm{~dB}$, the SINR loss is about $3.2 \mathrm{~dB}$ if $\epsilon=10^{-1}$, and $7.5 \mathrm{~dB}$ if $\epsilon=2 \times 10^{-1}$.

When the frequency offset is due to Condition 3 only, from (15), we have the following inequality

$$
\operatorname{Var}\left\{\hat{\varepsilon_{k}} \mid \mathbf{y}\right\}>\frac{1}{\alpha_{k} \cdot \mathrm{SNR}_{k}^{2}}+\frac{1}{\beta_{k} \cdot \mathrm{SNR}_{k}}+\frac{\pi^{2} \epsilon^{2}}{9 \varpi_{k}} .
$$

(21) can be restated as

$$
\epsilon>\sqrt{\frac{9 \varpi_{k}}{3 \varpi_{k}-\pi^{2}} \cdot\left(\frac{1}{\alpha_{k} \cdot \mathrm{SNR}_{k}^{2}}+\frac{1}{\beta_{k} \cdot \mathrm{SNR}_{k}}\right)} .
$$

(22) implies that a noisy OFDMA system is interferencelimited, and the carrier frequency offset compensation errors $\epsilon$ can be seen as a frequency instability in a noisy environment. In a static wireless channel with zero Doppler Shift, by using a carrier frequency offset estimator with a high accuracy, this frequency instability may become negligible at a high SNR. For example, when algorithm proposed in [2] is considered for each user, we have $\alpha_{k}=\varpi_{k}=4 \pi^{2} N$ and $\beta_{k}=2 \pi^{2} N$ for each $k$. With $N=1024, \epsilon$ is lower bounded by $1.5 \times 10^{-2}$ with $\mathrm{SNR}=0 \mathrm{~dB}$. For an $\mathrm{SNR}$ of $10 \mathrm{~dB}$, this lower bound is about $4 \times 10^{-3}$. In real systems, the residual offset $\epsilon$ will be mainly caused by Conditions 1 and 2 .

\section{CAPACITY OF OFDMA UPLINK WITH CARRIER FREQUENCY OFFSET}

For an OFDMA system with $K$ users simultaneously access a base station, the uplink ergodic capacity in a frequencyselective fading channel can be defined as [16, page 182]:

$$
\mathbb{C}_{U}=\max _{G_{1}, \cdots, G_{M}, P_{0}, \cdots, P_{N-1}} \sum_{i=0}^{N-1} \log \left(1+\gamma_{i}\right)
$$

(Bits/OFDMA Symbol), subject to

$$
\begin{cases}\sum_{m \in G_{k}} \log \left(1+\gamma_{m}\right) \geq R_{k} & 1 \leq k \leq K \\ \sum_{m \in G_{k}} P_{m}=\mathcal{N}_{k} \bar{P}_{k} & 1 \leq k \leq K\end{cases}
$$

where $\gamma_{m}$ stands for the SINR of subcarrier $m, R_{k}$ and $\bar{P}_{k}$ represent the rate of reliable communication and the averaged power constraint in user $k$, respectively. For a frequencyselective fading channel with Channel State Information at the receiver (CSIR), we have [16]

$$
\mathbb{C}_{U \mid C S I R} \cong \begin{cases}N \cdot \log _{2}^{e} \cdot \mathbb{E}\left\{\gamma_{i}\right\} & \text { Low SINR, } \\ N \cdot \mathbb{E}\left\{\log \left(\gamma_{i}\right)\right\} & \text { High SINR. }\end{cases}
$$

Note that the uplink capacity for an AWGN channel is $\mathbb{C}_{U \mid A W G N}=\sum_{i=0}^{N-1} \log \left(1+\mathrm{SNR}_{i}\right)$.

When the statistical information of the carrier frequency offsets in an OFDMA uplink transmission are known, the SINR of each subcarrier for each user can be calculated. By using a water-filling optimization, the power allocated to the $m$-th subcarrier of user $k$ is given by [16]:

$$
P_{m \in G_{k}}^{*}=\left(\frac{1}{\lambda_{k}}-\frac{\frac{\pi^{2} \epsilon^{2} \kappa_{m}}{9}+\sigma_{w}^{2}}{\left|H_{k}^{m}\right|^{2} \frac{\sin ^{2}\left(\pi \varepsilon_{k}\right)}{N^{2} \sin ^{2}\left(\frac{\pi \varepsilon_{k}}{N}\right)}}\right)^{+} \quad 1 \leq k \leq K,
$$

where $\sum_{k=1}^{K}\left(\lambda_{k} \sum_{m \in G_{k}} P_{m \in G_{k}}^{*}\right)=\sum_{k=1}^{K} \mathcal{N}_{k} \bar{P}_{k}$. Thus the uplink capacity is

$\mathbb{C}_{U \mid W F}=\sum_{k=1}^{K} \sum_{m \in G_{k}} \mathbb{E}\left\{\log \left(1+\frac{P_{m}\left|H_{k}^{m}\right|^{2} \frac{\sin ^{2}\left(\pi \varepsilon_{k}\right)}{N^{2} \sin ^{2}\left(\frac{\pi \varepsilon_{k}}{N}\right)}}{\frac{\pi^{2} \epsilon^{2} \kappa_{m}}{9}+\sigma_{w}^{2}}\right)\right\}$.

Fig. 2 illustrates channel capacity of the OFDMA uplink with carrier frequency offset and $N=1024$. A larger residual frequency offset $\epsilon$ results in a larger capacity losses. For example, in a frequency-selective fading channel with an SNR of $20 \mathrm{~dB}$ and CSIR, if the water-filling power allocation is not applied, an uplink capacity of 6000 (Bits/OFDMA Symbol) can be achieved when $\epsilon=0.01$. This capacity will reduced to about 5700 (Bits/OFDMA Symbol) when $\epsilon=0.05$, or to about 5000 (Bits/OFDMA Symbol) when $\epsilon=0.1$. By using water-filling power allocation method, the uplink capacity for $\epsilon=0.01, \epsilon=0.05$ and $\epsilon=0.1$ will be about 6100 (Bits/OFDMA Symbol), 5800 (Bits/OFDMA Symbol) and 5100 (Bits/OFDMA Symbol), respectively, i.e., capacity improvement of about 100 (Bits/OFDMA Symbol) is achieved for each scenario. More capacity improvement can be achieved by using a water-filling method as compared to CSIR at low SNR. For example, with $\mathrm{SNR}=10 \mathrm{~dB}$ and CSIR, the uplink capacity of about 3000 (Bits/OFDMA Symbol), 2950 (Bits/OFDMA Symbol) and 2800 (Bits/OFDMA Symbol) can be achieved for $\epsilon=0.01, \epsilon=0.05$ and $\epsilon=$ 0.1 , respectively, and the corresponding capacity can be 


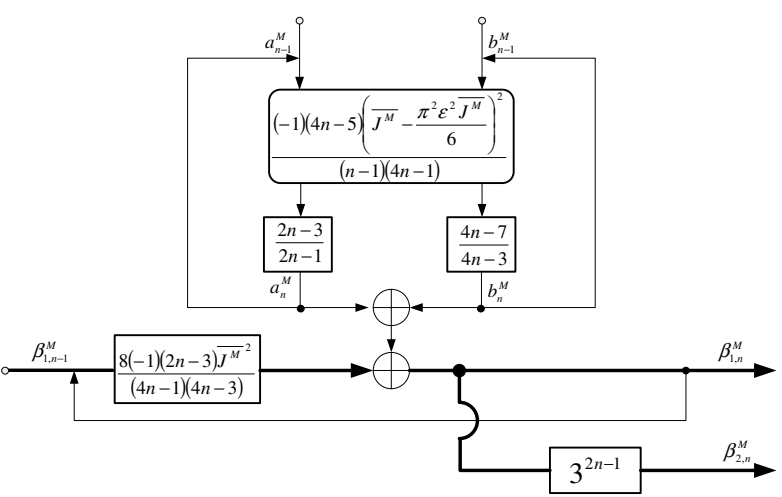

Fig. 3. The systematic illustration of coefficients' generation.

increased to about 3650 (Bits/OFDMA Symbol), 3600 (Bits/OFDMA Symbol) and 3500 (Bits/OFDMA Symbol), respectively.

\section{BER PERFORMANCE}

The BER as a function of the SINR for an OFDMA uplink is derived in this section. Here $M$-ary square QAM with Gray mapping is assumed to modulate each subcarrier. The BER performance for square QAM is discussed in [17], [18]. For adaptive power allocation (requires channel knowledge at each transmitter), we assume that the average received power for each user is identical. With CSIR, like in [17], the averaged BER for OFDMA uplink transmission is given by

$$
\begin{aligned}
& P_{B E R}^{M} \\
& \quad \cong \frac{\sqrt{M}-1}{\sqrt{M} \log _{2} \sqrt{M}} \cdot \mathbb{E}\left\{\operatorname{erfc}\left(\sqrt{\frac{3 \log _{2} M \cdot \gamma_{m \mid m \in G_{k}}}{2(M-1) \log _{2} M}}\right)\right\} \\
& +\frac{\sqrt{M}-2}{\sqrt{M} \log _{2} \sqrt{M}} \cdot \mathbb{E}\left\{\operatorname{erfc}\left(3 \sqrt{\frac{3 \log _{2} M \cdot \gamma_{m \mid m \in G_{k}}}{2(M-1) \log _{2}^{M}}}\right)\right\},
\end{aligned}
$$

where the expectation is with respect to $\gamma_{m \mid m \in G_{k}}$. It is difficult or impossible to represent $P_{B E R}^{M}$ in closed-form.

When the number of the users that access a base station is large enough, from [19, page 939], we know that $\operatorname{erfc}\left(\sqrt{\frac{3 \log _{2} M \cdot \gamma_{m \mid m \in G_{k}}}{2(M-1) \log _{2} M}}\right)$ and $\operatorname{erfc}\left(3 \sqrt{\frac{3 \log _{2} M \cdot \gamma_{m \mid m \in G_{k}}}{2(M-1) \log _{2}^{M}}}\right)$ can be represented as an infinite series, and, therefore, $P_{B E R}^{M}$ is given by

$$
\begin{aligned}
P_{B E R}^{M} & =\frac{2 \sqrt{M}-3}{\sqrt{M} \log _{2} \sqrt{M}}-\frac{2 \sqrt{6}(\sqrt{M}-1)}{\pi \epsilon \log _{2} \sqrt{M} \sqrt{M \pi \mathcal{J}^{M}}} \sum_{n=1}^{\infty} \beta_{1, n}^{M} \\
& -\frac{2 \sqrt{6}(\sqrt{M}-2)}{\pi \epsilon \log _{2} \sqrt{M} \sqrt{M \pi \mathcal{J}^{M}}} \sum_{n=1}^{\infty} \beta_{2, n}^{M},
\end{aligned}
$$

where $\beta_{1, n}^{M}=\frac{(-1)^{n+1}}{(2 n-1)(n-1) !} \int_{0}^{\pi \epsilon \sqrt{\frac{\bar{J}}{6}}}\left(\overline{\mathcal{J}}^{M}-y^{2}\right)^{2 n-1} \mathrm{~d} y$,

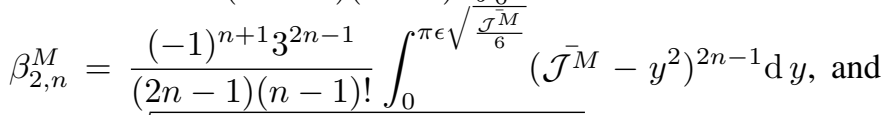
$\overline{\mathcal{J}^{M}}=\sqrt{\frac{3 \log _{2} M}{2(M-1) \log _{2}^{M}} \cdot \frac{\mathrm{SNR}}{\frac{\pi^{2} \epsilon^{2} \mathrm{SNR}}{9}+1}}$.

After numerical calculations, we have

$$
\begin{aligned}
\beta_{1, n}^{M}= & a_{n}^{M}+b_{n}^{M}-\frac{8(2 n-3) \overline{\mathcal{J}^{M}}}{(4 n-1)(4 n-3)} \cdot \beta_{1, n-1}^{M} \\
= & \frac{(-1)(2 n-3)(4 n-5)\left(\overline{\mathcal{J}^{M}}-\frac{\pi^{2} \epsilon^{2} \overline{\mathcal{J}}^{M}}{6}\right)^{2}}{(n-1)(2 n-1)(4 n-1)} \cdot a_{n-1}^{M} \\
& +\frac{(-1)(4 n-5)(4 n-7)\left(\overline{\mathcal{J}^{M}}-\frac{\pi^{2} \epsilon^{2} \mathcal{J}^{M}}{6}\right)^{2}}{(n-1)(4 n-1)(4 n-3)} \cdot b_{n-1}^{M} \\
& -\frac{8(2 n-3) \overline{\mathcal{J}}^{2}}{(4 n-1)(4 n-3)} \cdot \beta_{1, n-1}^{M},
\end{aligned}
$$

and

$$
\beta_{2, n}^{M}=3^{2 n-1} \beta_{1, n-1}^{M} .
$$

Given the initial condition of $a_{1}^{M}=$ $\frac{\pi \epsilon \sqrt{\frac{\mathcal{J}^{M}}{6}}\left(\overline{\mathcal{J}^{M}}-\frac{\pi^{2} \epsilon^{2} \overline{\mathcal{J}}^{M}}{6}\right)}{3}, \quad b_{1}^{M}=\frac{2 \pi \epsilon \mathcal{J}^{M} \sqrt{\frac{\mathcal{J}^{M}}{6}}}{3}$, $\beta_{1,1}^{M}=\left(\pi \epsilon-\frac{\pi^{3} \epsilon^{3}}{18}\right) \overline{\mathcal{J}^{M}} \sqrt{\frac{\overline{\mathcal{J}}^{M}}{6}} \quad$ and $\quad \beta_{2,1}^{M}=$ $3\left(\pi \epsilon-\frac{\pi^{3} \epsilon^{3}}{18}\right) \overline{\mathcal{J}^{M}} \sqrt{\frac{\overline{\mathcal{J}^{M}}}{6}}$, we can easily generate $a_{n>1}^{M}$, $b_{n>1}^{M}, \beta_{1, n>1}^{M}$ and $\beta_{2, n>1}^{M}$, as demonstrated in Fig. 3 .

In the real systems, when we approximate $P_{B E R}^{M}$ by using (29), an infinite series is usually impractical. In order to evaluate the validity of (29) with a finite order series in approximating (28), we test different orders in (29) and evaluate its convergence to (28), as shown in Fig. 4. With a small order, (29) approximates $P_{B E R}^{M}$ for each constellation well at a low SNR, and a deviation appears between them as SNR increases. This deviation appears earlier for a smaller $\epsilon$. For example, when each subcarrier is modulated by QPSK, with $\epsilon=0.01$ and an order of $10,(29)$ converges at $P_{B E R}^{M}$ only when $\mathrm{SNR} \leq 7 \mathrm{~dB}$. With $\epsilon=0.1$, this convergence can be achieved well until $\mathrm{SNR}=8 \mathrm{~dB}$. A similar convergent performance also holds for a high-order modulation, e.g., 16QAM. This deviation can be mitigated by increasing the order. For example, by increasing the order to 50, (29) with each modulation converges at $P_{B E R}^{M}$ well at $\mathrm{SNR}=10 \mathrm{~dB}$ for each evaluated $\epsilon$.

The BER performance losses due to the carrier frequency offsets are shown in Fig. 5, where 16 users access a base station simultaneously, and the number of subcarriers allocated to each user is identical. The number of subcarriers is 1024. Each subcarrier is modulated by using either QPSK or 16QAM both with Gray bit mapping. During multiple transmissions, each user's modulation scheme remains unchanged. The increase in 


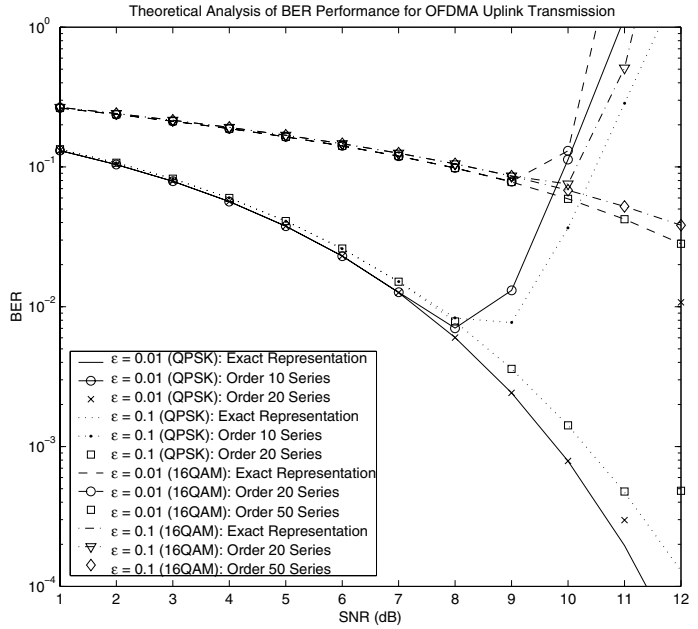

Fig. 4. The approximation of BER performance with finite order series.

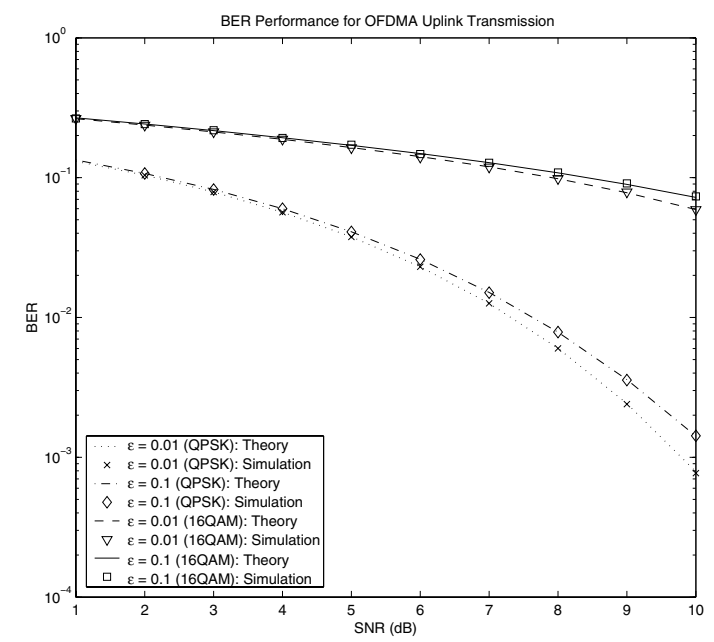

Fig. 5. BER performance with non-zero carrier frequency offset.

frequency offset $\epsilon$ increases the BER regardless of the modulation format. For example, with QPSK-modulated subcarriers, the BER performance loss amounts to about $0.5 \mathrm{~dB}$ when the frequency offset increases from $\epsilon=0.01$ to $\epsilon=0.1$. The corresponding loss for 16QAM is $1 \mathrm{~dB}$. For a same SINR, since the effective $\frac{E_{b}}{N_{0}}$ in 16QAM is much smaller than that in QPSK ( $E_{b}$ and $N_{0}$ represent the energy per bit and the spectrum density of additive noise, respectively), the BER for 16QAM is much higher than that for the latter.

\section{CONCLUSIONS}

In this paper, the performance degradation due to the carrier frequency offset in an OFDMA uplink, which is interferencelimited, is analyzed. Both the uplink capacity and the BER will be degraded by the residual carrier frequency offsets. Using the statistical information of frequency offset and CSI, we derived a water-filling power allocation method to improve the uplink capacity for each user. We also derived an infinite-series BER expression, and its coefficients for different orders can be iteratively calculated, making this approximation tractable in the real systems.

\section{REFERENCES}

[1] I. Koffman and V. Roman, "Broadband wireless access solutions based on OFDM access in IEEE 802.16," IEEE Communications Magazine, vol. 40, no. 4, pp. 96-103, Apr. 2002.

[2] P. Moose, "A technique for orthogonal frequency division multiplexing frequency offset correction," IEEE Trans. Commun., vol. 42, no. 10, pp. 2908-2914, Oct. 1994.

[3] L. Rugini and P. Banelli, "BER of OFDM systems impaired by carrier frequency offset in multipath fading channels," IEEE Transactions on Wireless Communications, vol. 4, no. 5, pp. 2279-2288, Sept. 2005.

[4] T. Schmidl and D. Cox, "Robust frequency and timing synchronization for OFDM," IEEE Trans. Commun., vol. 45, no. 12, pp. 1613-1621, Dec. 1997.

[5] M. Morelli and U. Mengali, "An improved frequency offset estimator for OFDM applications," IEEE Commun. Lett., vol. 3, no. 3, pp. 75-77, Mar. 1999.

[6] Z. Zhang, W. Jiang, H. Zhou, Y. Liu, and J. Gao, "High accuracy frequency offset correction with adjustable acquisition range in OFDM systems," IEEE Trans. Wireless Commun., vol. 4, no. 1, pp. 228-237, Jan. 2005.

[7] J. Choi, C. Lee, H. W. Jung, and Y. H. Lee, "Carrier frequency offset compensation for uplink of OFDM-FDMA systems," IEEE Commun. Lett., vol. 4, no. 12, pp. 414-416, Dec. 2000.

[8] M. Morelli, "Timing and frequency synchronization for the uplink of an OFDMA system," IEEE Trans. Commun., vol. 52, no. 2, pp. 296-306, Jan. 2004.

[9] Z. Cao, U. Tureli, and Y.-D. Yao, "Deterministic multiuser carrierfrequency offset estimation for interleaved OFDMA uplink," IEEE Trans. Commun., vol. 52, no. 9, pp. 1585 - 1594, Sept. 2004.

[10] H. Bolcskei, "Blind high-resolution uplink synchronization of OFDMbased multiple access schemes," in Signal Processing Advances in Wireless Communications, 1999. SPAWC '99. 1999 2nd IEEE Workshop on, Annapolis, MD, 1999, pp. 166-169.

[11] M.-O. Pun, M. Morelli, and C.-C. Kuo, "Maximum-likelihood synchronization and channel estimation for ofdma uplink transmissions," IEEE Trans. Commun., vol. 54, no. 4, pp. 726-736, Apr. 2006.

[12] D. Huang and K. Letaief, "An interference-cancellation scheme for carrier frequency offsets correction in OFDMA systems," IEEE Trans. Commun., vol. 53, no. 7, pp. 1155-1165, July 2005.

[13] K. Kim, Y. Han, and S.-L. Kim, "Joint subcarrier and power allocation in uplink OFDMA systems," IEEE Commun. Lett., vol. 9, no. 6, pp. 526-528, June 2005.

[14] J. Jang and K. B. Lee, "Transmit power adaptation for multiuser OFDM systems," IEEE J. Select. Areas Commun., vol. 21, no. 2, pp. 171-178, Feb. 2003.

[15] S. Haykin, Adaptive Filter Theory. New Jersey: Prentice-Hall, 1996.

[16] D. Tse and P. Viswanath, Fundamentals of Wireless Communication. Cambridge University Press, 2005.

[17] K. Cho and D. Yoon, "On the general BER expression of one- and twodimensional amplitude modulations," IEEE Trans. Commun., vol. 50, no. 7, pp. 1074-1080, July 2002.

[18] L.-L. Yang and L. Hanzo, "A recursive algorithm for the error probability evaluation of m-QAM," IEEE Commun. Lett., vol. 4, no. 10, pp. 304306, Oct. 2000.

[19] I. S. Gradshteyn and I. M. Ryzhik, Table of Integrals, Series, and Products, 5th ed. Academic Press, Inc., 1994. 